\title{
Summary.
}

I. The reaction of an excess of phenyl-magnesium bromide upon the methyl or ethyl ester of phthalic acid gives a mono-hydric tertiary alcohol which is a derivative of furfurane.

2. If the product of the reaction of an excess of phenyl-magnesium bromicle upon the esters of phthalic acid is heated to about $300^{\circ}$, it loses a molecule of water and gives the compound $\mathrm{C}_{26} \mathrm{H}_{18} \mathrm{O}$ of m. p. $192-193^{\circ}$ which Shibata ${ }^{1}$ claimed to be a derivative "of phthalan and the direct product of the original condensation.

Houston, Tex.

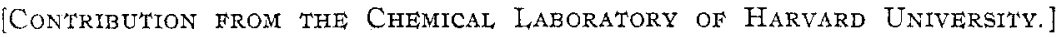

\section{ADDITION REACTIONS OF PHOSPHORUS HALIDES. I. THE MECHANISM OF THE REACTION OF THE TRI- CHLORIDE WITH BENZALDEHYDE.}

By James B. Conant and Alexander D. Macdonard. Received August 11, 1920。

\section{Introduction.}

It has been shown in a previous paper ${ }^{2}$ that phosphorus trichloride adds to the ends of the conjugated system of certain unsaturated ketones. This addition takes place with an increase in valence of the phosphorus atom from 3 to 5 . The chlorine atoms in the addition product are very active and are readily replaced by hydroxyl or oxygen when the reaction mixture is treated with acetic acid or acetic anhydride. The product thus formed is a phosphonic acid or an intermediate cyclic compound. An exactly similar reaction takes place when simple aldehydes or ketones are treated with phosphorus trichloride. In this case, however, the primary addition product is formed by the 1,2 -addition of the phosphorus atom.

$$
\begin{aligned}
& \mathrm{RCHO}+\mathrm{PCl}_{3} \rightleftharpoons \mathrm{RCHO} \\
& \checkmark \\
& \mathrm{PCl}_{3}
\end{aligned}
$$

(I).

Benzaldehyde was chosen as a suitable aldehyde for studying the mechanism of this reaction and only the results obtained with this substance are presented here. The reaction, however, takes place readily with most aldehydes and ketones and the limits of the reaction will be discussed in a subsequent paper.

\section{The Primary Reversible Reaction.}

When benzaldehyde and phosphorus trichloride are brought together consiclerable heat is evolved and the addition reaction (Equation I) proceeds to a definite equilibrium. If equimolecular amounts of the 2 sub-

1 Loc. cit.

2 'THIS JOURnal, 42, 830 (1920). 


\section{$233^{8}$ JAMES B. CONANT AND ALEXANDGR D. MACDONALD.}

stances are employed, only about $30 \%$ of the addition product is formed. Or treating such a reaction mixture with water both the unused trichloride and the addition product react vigorously. The former produces phosphorous acid, the latter an $\alpha$-hydroxy-phosphonic acid as the final product.

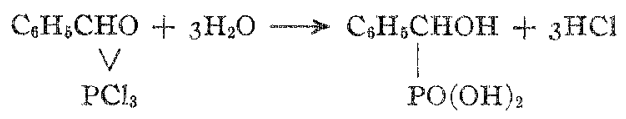

The amount of phosphorous acid thus formed can be determined by caun tiously oxidizing it to phosphoric acid and precipitating it in the usual manner with magnesia mixture. The hydroxy phosphonic acid does not produce a precipitate under the same conditions. The amount of trichloride in the equilibrium mixture can thus be easily ascertained.

Quantitative experiments of this sort were performed with varying ratios of the 2 substances and also with varying amounts of an inert sol vent (benzene). The equilibrium constant

$$
K=\frac{\left[\mathrm{C}_{6} \mathrm{H}_{5} \mathrm{CHO}\right]\left[\mathrm{PCl}_{3}\right]}{\left.V\left[\mathrm{C}_{6} \mathrm{H}_{5} \mathrm{CHOPCl}\right)_{3}\right]}
$$

could thus be calculated from the concentration as determined in each experiment. Samples taken from time to time showed that a condition of equilibrium was usually reached in the course of 28 hours. The values of $K$ for 7 different experiments varied from 6.9 to 8.3 ; the average was 7.4. The initial concentrations of the reacting substances were varied over wide limits in the different experiments. The values for $K$ are constant within the experimental errors of the method. They clearly show that the primary addition reaction is reversible and can be expressed in terms of the usual law of mass action.

\section{Intermediate Products Containing a Phosphorus Oxygen Ring.}

The addition product reacts with acetic anhydride and, as in the case of the unsaturated ketones, "he products are acetyl chloride and a mixture of an acid chloride (I) and an anhydride (II). Phosphorus trichloride reacts very slowly with acetic anhydride below $50^{\circ}$, the primary addition compound reacts rapidly. "The equilibrium of the initial addition reaction is thus upset by the removal of the product and the entire set of reactions proceed to completion. When a large excess of acetic anhydride is used, most of the acid chloride first formed is converted into the acid anhydride. These reactions are represented by the following equations.

$$
\begin{array}{cc}
\mathrm{C}_{6} \mathrm{H}_{5} \mathrm{CHO} \\
\underset{P \mathrm{Cl}_{3}}{V}+\left(\mathrm{CH}_{3} \mathrm{CO}\right)_{2} \mathrm{O}= & \mathrm{C}_{6} \mathrm{H}_{5} \mathrm{CHO}+{ }_{2} \mathrm{CH}_{3} \mathrm{COCl} \\
& \text { POCl }
\end{array}
$$

Toe.cit.

(1). 


$$
{ }_{2} \mathrm{C}_{6} \mathrm{H}_{5} \mathrm{CHO}+\left(\mathrm{CH}_{3} \mathrm{CO}\right)_{2} \mathrm{O}=\left(\begin{array}{c}
\mathrm{C}_{6} \mathrm{H}_{5} \mathrm{CHO} \\
V \\
\mathrm{PCl}_{3}
\end{array}\right)_{2}^{\mathrm{O}}+{ }_{2} \mathrm{CH}_{3} \mathrm{COCl}
$$

The mixture of the acid chloride and anhydride is left as a gum after the evaporation of the acetyl chloride and the excess of acetic anhydride. This gum, when treated with water, yields hydrochloric acid and a monobasic acid containing a phosphorus oxygen ring. The amount of chloride ion thus formed is a measure of the relative amount of acid chloride (I) in the mixture.

$$
\begin{array}{cc}
\mathrm{C}_{6} \mathrm{H}_{5} \mathrm{CHO} & +\mathrm{H}_{2} \mathrm{O} \\
\underset{\text { POCl }}{V} & =\mathrm{C}_{6} \mathrm{H}_{5} \mathrm{CHO}+\mathrm{HCl} \\
V & \text { POOH }
\end{array}
$$

This ring acid was isolated in the form of an insoluble barium salt. Its structure follows from the fact that it is a monobasic acid which, on boiling with water, is converted into the hydroxy-phosphonic acid.

$$
\begin{array}{cc}
\mathrm{C}_{6} \mathrm{H}_{5} \mathrm{CHO}+\mathrm{H}_{2} \mathrm{O} & =\mathrm{C}_{6} \mathrm{H}_{5} \mathrm{CHOH} \\
\mathrm{POOH} & \mathrm{PO}(\mathrm{OH})_{2}
\end{array}
$$

It reduces potassium permanganate solution slowly, whereas the hydroxyphosphonic acid is oxidized immediately by the same reagent. This difference would, of course, be expected from the structure of the 2 compounds. The isolation of this acid proves that the fundamental process in the interaction of benzaldehyde and phosphorus trichloride is the $T, 2$ addition of the phosphorus atom to the carbonyl group.

Hitherto no acid containing a phosphorus oxygen ring has been reported. It is suggested that the name "phostonic" be applied to the general class of such acids from their obvious similarity to lactonic acids. The individual substances are named in this article, however, as inner esters by prefixing anhydro-; the acid in question is thus anhydro- $\alpha$-hydroxybenzylphosphoric acid.

It is interesting to compare the stability of the phosphorus oxygen ring in this acid with the stability of the ring in the intermediate products obtained from unsaturated ketones. In the case of the unsaturated ketones it was shown that the intermediate products contained the 5 -membered unsaturated ring: $-\mathrm{C}-\mathrm{C}=\mathrm{C}-$. This ring was so easily

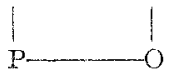

opened by water and acid that only the anhydride and acid chloride could be obtained. The phostonic acid, however, is only slowly converted into the hydroxyphosphonic acid by boiling with pure water. The reaction is accelerated in aqueous solution by mineral acids. In acetic acid the ring is opened rather slowly by hydrogen chloride. The difference between these 2 rings is thus very great and is an exception to 
the usual belief in regard to the greater stability of 5 -membered rings. This may be due, of course, to the fact that one ring contains an ethylene linkage and the other does not.

\section{The Reaction in Glacial Acetic Acid.}

Fossek ${ }^{1}$ prepared a number of hydroxy-phosphonic acids by treating phosphorus trichloride with a large excess of an aldehyde or ketone and decomposing the resulting oil with water. He suggested no mechanism for the reaction ${ }^{2}$ but clearly showed that his products were $\alpha$-hydroxyphosphonic acids. The necessity of employing a large excess of one of the substances is now obvious. Since water was tised to decompose the reaction mixture there was obtained only an amount of phosphonic acid corresponding to the equilibrium of the initial reaction; by the use of the large excess of aldehyde, the reaction was forced as far to the tight as possible. It is possible to complete the reaction with equimolecular amounts by the use of acetic anhydride for the reasons given above. To obtain the hydroxy-phosphonic acid, the product, of course, must be boiled with water to decompose the intermediate phostonic acid.

Glacial acetic acid also reacts more rapidly with the addition product than with the trichloride. However, since the trichloride reacts rapidly with acetic acid on warming, it is necessary to work below $35^{\circ}$ and it is best to allow the 2 compounds to react and then add the acid. Here also the phostonic acid is formed as in the case of acetic anhydride. If the reaction mixture is allowed to stand for several days this is slowly converted into the hydroxy-phosphonic acid. Saturation of the mixture with hydrogen chloride hastens the process. This reaction proceeds probably through the formation of the acid chloride of the hydroxy-phosphonic acid, which then reacts with the acetic acid.

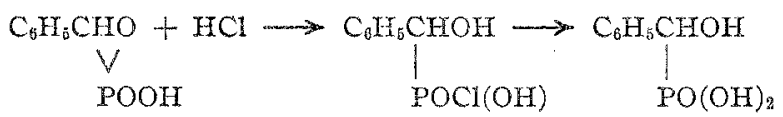

If the mixture is poured into water and the solution evaporated to dryness the conversion is rapid and complete. This is the most satisfactory method of preparing the hydroxy-phosphonic acid. Good yields can be obtained without using an excess of either of the 2 substances. As the hydroxy-phosphonic acid is very soluble in water it is somewhat hard to isolate in a pure condition. It was found that an aniline salt of the acid could be readily prepared in ether solution and was easily recrystallized from alcohol. By the use of this salt the acid can be quickly and conveniently isolated.

${ }^{1}$ Fossek, Monatsh., 5, I20, 627 (1884); 7, I2x (1886).

a Michaelis, Ber., 18, 899 (1895); $i b i d_{0}, 3, \times 297$ (ت90r), referring to Fosseir's work suggested that an intermediate ring compound is first formed in this and similar cases. 


\section{Experimental.}

\section{Equilibrium Measurements.}

A mixture of $5.0 \mathrm{~g}$. of benzaldehyde $(4.78 \mathrm{cc}$.) and $6.48 \mathrm{~g}$. of phosphorus trichloride $(4.06 \mathrm{cc}$.) was placed in a tightly stoppered Erlenmeyer flask and allowed to stand at room temperature $\left(25^{\circ}\right)$. One-cc. samples were removed by a pipet at intervals and introduced into $5^{\circ} \mathrm{cc}$. of distilled water; the whole was then shaken vigorously. The aqueous solution was extracted with two $20-\mathrm{cc}$. portions of benzene to remove unchanged benzaldehyde and then heated on the steam-bath for a few minutes to remove any traces of benzene. Bromine water was added a little at a time to the solution after it had been cooled to room temperature. The phosphorous acid formed by the interaction of the trichloride and water was thus oxidized to phosphoric acid; the addition or bromine water was continued until a permanent color was produced. Magnesia mixture was now added and the magnesium ammonium phosphate allowed to precipitate in the usual way. The precipitate was filtered, washed, and ignited to constant weight. Parallel experiments with pure hydroxybenzyl phosphonic acid showed that it did not produce a precipitate under these conditions. Similar experiments were performed with various proportions of the 2 substances and also with the reaction mixture diluted with anhydrous benzene to $25 \mathrm{cc}$, $50 \mathrm{cc}$., $75 \mathrm{cc}$., and Ioo cc., respectively.

Tabie 1.-VALUes of $K$ from the Final Measurements.

\begin{tabular}{|c|c|c|c|c|c|c|c|}
\hline Expt. & $\begin{array}{l}\text { Mols. of } \\
\mathrm{CeH}_{3} \mathrm{CHO} \\
a .\end{array}$ & $\begin{array}{c}\text { Mols of } \\
\mathrm{PCi}_{3=} \\
\text { b. }\end{array}$ & $\begin{array}{c}\mathrm{Mg}_{2} \mathrm{P}_{2} \mathrm{O}_{7} \\
\text { equiv, } \\
\text { to } \mathrm{PCl}_{8} \\
\text { in } 1 \mathrm{cc} . \\
\mathrm{G} .\end{array}$ & $\begin{array}{c}\text { Actual wt. } \\
\mathrm{Mg}_{2} P_{2} \mathrm{O}_{7} \\
\text { at equil. } \\
\mathrm{C} .\end{array}$ & $\begin{array}{c}\text { Fract. of } \\
\text { PCis } \\
\text { reacted. } \\
y .\end{array}$ & $\begin{array}{l}V \text { in } \\
\text { Liters. }\end{array}$ & $K$ \\
\hline I........ & 0.0472 & 0.0472 & 0.595 & 0.412 & 0.308 & 0.00884 & 8.3 \\
\hline $2 \ldots \ldots \ldots$ & 0.0472 & 0.0944 & $0.8 I 7$ & 0.646 & 0.209 & 0.0129 & 8.0 \\
\hline $3 \ldots \ldots \ldots$ & 0.0944 & 0.0472 & 0.387 & $0.22 \mathrm{I}$ & 0.429 & 0.0136 & $7 \cdot 3$ \\
\hline $4 \ldots \ldots \ldots$ & 0.0944 & 0.0944 & 0.422 & 0.305 & 0.277 & 0.025 & $7 \cdot I$ \\
\hline $5 \ldots \ldots \ldots$ & 0.0944 & 0.0944 & $0.2 I I$ & 0.173 & 0.182 & 0.050 & 6.9 \\
\hline $6 \ldots \ldots$ & 0.0944 & 0.0944 & $0.14 I$ & 0.123 & o. 129 & 0.075 & $7 \cdot 4$ \\
\hline $7 \ldots \ldots$ & 0.0944 & 0.0944 & 0.1055 & 0.094 & 0.109 & $\begin{array}{l}\text { o. noo } \\
\text { Average, }\end{array}$ & $\begin{array}{l}6.9 \\
7.4\end{array}$ \\
\hline
\end{tabular}

\begin{tabular}{|c|c|c|c|c|c|c|c|}
\hline \multirow[b]{2}{*}{ Expt. } & \multirow{2}{*}{$\begin{array}{l}\mathrm{Mg}_{2} \mathrm{P}_{2} \mathrm{O} \\
\text { equiv, to } \\
\mathrm{PC1} \text { in } \\
1 \mathrm{ce} .\end{array}$} & \multicolumn{6}{|c|}{ Weights of $\mathrm{Mg}_{2} \mathrm{P}_{2} \mathrm{O}_{7}(W)$ formed and fract. of $\mathrm{PCl}_{3}$ reacted $(y)$. } \\
\hline & & \multicolumn{2}{|c|}{3 hours. } & \multicolumn{2}{|c|}{$W .28$ houts. $y$} & $W$ & $y$. \\
\hline I.... & 0.595 & 0.433 & 0.272 & 0.412 & 0.308 & & \\
\hline $2 \ldots \ldots$ & 0.817 & 0.670 & 0.180 & 0.646 & 0.209 & & \\
\hline \multirow[t]{2}{*}{$3 \ldots$} & 0.387 & 0.279 & 0.279 & $0.22 \mathrm{I}$ & 0.429 & & \\
\hline & & \multicolumn{2}{|c|}{4.7 hours. } & \multicolumn{2}{|c|}{71 hours. } & \multicolumn{2}{|c|}{122 hours. } \\
\hline $4 \ldots$. & 0.422 & 0.338 & 0.199 & 0.318 & 0.246 & 0.305 & 0.277 \\
\hline $5 ., \ldots$ & $0.2 I I$ & 0.179 & O. I 5 I & 0.176 & o. I 66 & 0.173 & 0.182 \\
\hline $6 \ldots$ & $0.14 I$ & 0.123 & 0.129 & 0.120 & 0.147 & 0.123 & 0.129 \\
\hline $7 \ldots$ & 0.106 & 0.097 & 0.076 & 0.094 & 0.109 & 0.094 & 0.109 \\
\hline
\end{tabular}


The results obtained are summarized in Tables $I$ and II. Table I shows the values for $K$ calculated from the final measurements; Table II, the course of the reaction to the final equilibrium. The amount of mag. mesitum pyrophosphate equivalent to the trichloride initially present in onewec, of the reaction mixture could be calculated from the quatsity of materials used and the total volume. The difference between this value and the magnesium pyrophosphate actually weighed represents the amount of trichloride which had formed the addition product. The ratio of this difference to the initial amount is obviously the sraction of the trichloride reacted. If we represent this fraction by $y$, and the initial quantities (in mols.) of $\mathrm{C}_{6} \mathrm{H}_{5} \mathrm{CHO}$ by $a$ and of trichloride by $b$, the quantity of the addition product is $y b$. The equation for $K$ cats then be written as follows, where $V$ equals the volume in liters of the reaction mixture.

$$
K=\frac{(a-y b)(b-y b)}{V y b} .
$$

This method of determining $K$ is obviously not very accurate and the values obtained vary considerably. Nevertheless they are sufficiently constant to indicate the fundamental fact, namely, that the reaction under investigation comes to a definite equilibrium which is determined by the factors expressed in the ustal law of mass action. Similar results were obtained earlier in the research by a different method. The rereaction mixture was decomposed with water and the amount of unchanged benzaldehyde was determined by measuring its volume in a specially constructed pipet somewhat similar to that used in the Babcock nethod for determining fat in milk. These approximate values showed that the reaction came to an equilibrium in 2 to 3 hours (no solvent was employed, so that the experiments were parallel to No. $I$ in the tables). The fraction of benzaldehyde which reacted was 0.35 as compared with 0.30 determined by weighing the magnesium pyrophosphate.

\section{Reaction in Acetic Anhydride.}

A mixture of the acid chloride and anhydride of the phostonic acid was prepared bo the interaction of phosphorus trichloride, benzaldehyde, and acetic anhydride, A typical experiment follows, to g. of benzaldehyde (0.094 mol.), $\mathrm{r} 3 \mathrm{~g}$. of trichloride ( $0.094 \mathrm{~mol}$ ) , and $20 \mathrm{~g}$. of acetic axhydride $\left(0.20 \mathrm{~mol}\right.$ ) were mixed in a $\mathrm{I}_{5} \mathrm{O}-\mathrm{cc}$. distilling flask provided with a calcium chloride tube. The color of the mixture became pink and the temperature rose somewhat, but was kept below $40^{\circ}$, by cooling the flask with water. The mixture was allowed to stand overnight at room temperature. The contents of the flask were evaporated at a pressure of $50-60 \mathrm{~mm}$. at $50^{\circ}$ for 2 hours. A viscous resin weighing 22 $\mathrm{g}$. was left. This resin was soluble in chloroform, but no crystalline 
solid could be obtained from it. By making the chloroform solution of a known volume and taking definite amounts of it for various experiments, all the results could be calculated on the basis of the entire weight of materials employed.

Five such experiments were performed with various amounts of acetic anhydride. The proportion of acid chloride in the restlting mixture was determined in a typical experiment as follows. Two cc. of the chloroform solution of the resin (total vol. $=48 \mathrm{cc}$.) was evaporated to dryness and the residue dissolved in water. This solution was then acicified with nitric acid and titrated against $0.2 \mathrm{~N}$ silver nitrate according to Volhard's method. Equivalent of chloride ion found $=0.000234$, calculated for the acid chloride $=0.0039 \mathrm{r}$; therefore, the resin is $6 \%$ acid chloride. The experiments may be summarized as follows. Equimolecular amounts of benzaldehyde and trichloride were used in each experiment.

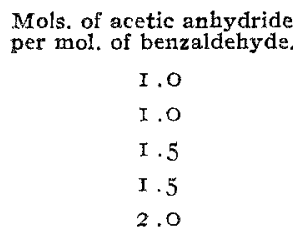

TABLE III,

Increasing the amount of acetic anhydride decreases the amount of acid chloride; this latter substance evidently reacts with the acetic anhydride to form acetyl chloride and the anhydride of the phostonic acid.

Aniline Salt of Hydroxybenzyl Phosphonic Acid, $\mathrm{C}_{6} \mathrm{H}_{5} \mathrm{CHOHPO-}$ (OH) $\mathrm{ONH}_{3} \mathrm{C}_{6} \mathrm{H}_{5}$. - A portion of the resin obtained in each of the 5 experiments just mentioned was converted into the aniline salt of hydroxy benzylphosphonic acid. For example, $5 \mathrm{cc}$. of the chloroform solution (total vol. $=48 \mathrm{cc}$.) was evaporated and the residue boiled with water for several hours and evaporated to dryness. The somewhat gurnmy residue of hydroxy-phosphonic acid was dissolved in a little alcohol and the solution diluted with 3 times its volume of ether. A slight excess of an ethereal solution of aniline was added. A voluminous white precipitate formed at once and was filtered off, washed with a mixture of ether and alcohol and dried. Weight $2.3 \mathrm{~g}$., which is equivalent to 22 . I g. for the entire mixture, or $84.5 \%$ of the calculated yield from $0.094 \mathrm{~mol}$. of aldehyde, which is $26.4 \mathrm{~g}$. Similar yields were obtained in the other 4 experiments. The resin was thus shown to be an intermediate stage in the formation of the hydroxy-phosphonic acid.

The aniline salt of hydroxybenzyl phosphoric acid could be recrys. tallized from alcohol. It melted with decomposition at $201-202^{\circ}$.

Calc, for $\mathrm{C}_{13} \mathrm{H}_{36} \mathrm{O}_{4} \mathrm{NP} ; \mathrm{N}, 5 . \mathrm{O}$, Found: $5 . \mathrm{X}$. 
The pure acid could be obtained from the salt as follows. Five g. was decomposed with $150 \mathrm{cc}$. of $5 \%$ solution of sodium hydroxide and the mixture distilled with steam until no more aniline came over. The residual solution was cooled, neutralized with acetic acid, and an excess of lead acetate added. A heavy white precipitate of the lead salt formed; this was filtered, washed with water, suspended in $200 \mathrm{cc}$. of water and decomposed with hydrogen sulfide. The lead sulfide was filtered off and the filtrate evaporated to dryness. $2.9 \mathrm{~g}$. of hydroxybenzyl phosphonic acid, $m$. p. $170-172^{\circ}$, was thus obtained. This acid was identical with material obtained from the reaction in acetic acid (see below) and recrystallized from dil. hydrochloric acid. It is identical in its properties with the substance prepared by Fossek. ${ }^{1}$

Barium Salt of Anhydro- $\alpha$-hydroxy-benzyl Phosphonic Acid, $\left(\mathrm{C}_{6} \mathrm{H}_{6} \mathrm{CHPOO}\right)_{2} \mathrm{Ba}$. - $20 \mathrm{~g}$. of benzaldehyde, $26 \mathrm{~g}$. of phosphorus trichloO

ride, and $29 \mathrm{~g}$. of acetic anhydride were mixed in a $150 \mathrm{cc}$. distilling flask and allowed to react as described in the previous experiments. After standing for 2 days at room temperature the mixture was evaporated under diminished pressure at a temperature of $40-45^{\circ}$. Forty g. of resin was thus obtained. Twenty-seven $\mathrm{g}$. of this resin was dissolved in $250 \mathrm{cc}$. of water by very gentle warming and stirring. The cooled solution was treated with the calculated quantity of barium hydroxide $(20 \mathrm{~g}$.) dissolved in $250 \mathrm{cc}$. of water. A white precipitate was formed. The solution was now made barely alkaline to litmus with ammoniurn hydroxide; the precipitate increased in amount. The solution was filtered and the precipitate washed with water and dried. The yield was $18.8 \mathrm{~g}$, which is $63 \%$ of the amount theoretically possible calculated from the amount of resin taken and the benzaldehyde initially employed.

Samples of barium salt thus prepared were dried to constant weight at $110^{\circ}$ and analyzed for barium and phosphorus. They were found, however, to be contaminated with barium phosphate. The phosphoric acid was undoubtedly produced by a side reaction between the trichloride and the acetic anhydride. The amount of phosphate present was determined and the analyses corrected for it. A sample of the salt was heated with dil. hydrochloric acid on the steam-bath until a clear solution was obtained. Magnesia mixture was added to the cooled solution and the magnesium ammonium phosphate precipitated by making the solution alkaline with ammonia in the usual way. The precipitate was contaminated with barium and was redissolved in nitric acid and the phosphate precipitated with ammonium molybdate. The ammonium phosphomolybdate was filtered, redissolved, and the phosphate precipitated as

1 Loc, cit. 
magnesium ammonium phosphate and ignited and weighed as the pyrophosphate.

Subs. (I), $0.3807,0.301 \mathrm{x}: \mathrm{BaSO}_{4}, 0.2064,0.1633 ; \mathrm{Mg}_{2} \mathrm{P}_{2} \mathrm{O}_{7}, 0.16 \mathrm{II}, 0.1238$.

Subs, 0.3400 , analyzed as above for phosphate: $\mathrm{Mg}_{2} \mathrm{P}_{2} \mathrm{O}_{7}$, o.orII.

Subs. (II), $0.3427,0.3437: \mathrm{BaSO}_{4}$, 0.1910, $0.1922 ; \mathrm{Mg}_{2} \mathrm{P}_{2} \mathrm{O}_{7}, 0.1453,0.1489$.

Subs., 0.3253 , analyzed for phosphate: $\mathrm{Mg}_{2} \mathrm{P}_{2} \mathrm{O}_{7}$, o.0120.

\begin{tabular}{|c|c|c|c|c|c|c|c|c|c|}
\hline \multirow{3}{*}{$\%$} & \multicolumn{5}{|c|}{ Found: Uncorrected. } & \multicolumn{4}{|c|}{ Corrected for phosphate. } \\
\hline & Calc. for & & & & & & & & r. \\
\hline & 28.9 & 31.9 & $3 I .9$ & 32.8 & 32.9 & 28.7 & 28.7 & 29.2 & 29.4 \\
\hline $\mathrm{P}$ & . 13.0 & I 1.8 & II.9 & I 1.8 & I2.I & I2. I & I2.I & 12.1 & $\mathrm{I} 2.4$ \\
\hline
\end{tabular}

The barium salt of anhydro- $\alpha$-hydroxy-benzyl phostonic acid is a white powder only slightly soluble in water, but readily soluble in dilute acids. It could not be recrystallized successfully. As would be expected from its structure, the salt is only very slowly oxidized by potassium permanganate solution. A comparison of the rate of oxidation of this compound and the neutral barium salt of hydroxy-phenyl phosphonic acid was made by shaking $0.1 \mathrm{~g}$. of the solids with $10 \mathrm{cc}$. of a very dilute pernamganate solution made slightly acid with sulfuric acid. The permanaganate color disappeared in 5 minutes in the case of the hydroxy-phenyl phosphonic salt, but faded only at the end of an hour with the phostonic acid salt.

Anhydro- $\alpha$-hydroxy-benzyl Phosphonic Acid.-This acid was obtained as a gum contaminated with barium salts by acidifying the barium salt. Seven g. of barium salt was placed in a mortar and ground with $50 \mathrm{cc}$. of $6 N$ hydrochloric acid. The mixture became pasty, and a white sticky gum separated on the sides of the mortar and on the pestle. The aqueous solution was poured off and the gum ground with 3 portions of cold water. It was then spread on a porous plate and dried in a desiccator over sulfuric acid. $2.6 \mathrm{~g}$. of dried material was thus obtained. When dissolved in alcohol it left a small amount of insoluble barium salt and the alcoholic solution on evaporation yielded a somewhat purer resin. However, all the barium compounds could not be removed by this treatment. An analysis showed that it still contained about 10\% of barium, presumably as the unchanged barium salt. The resin dissolved in sodium hydrogen carbonate with evolution of carbon dioxide.

The acid could be obtained only in this impure condition. However, the titration with sodium hydroxide, oxidation with permanganate, reaction with aniline, and hydrolysis to hydroxy-benzyl phosphonic acid, showed that the material at hand was the phostonic acid.

Subs., $0.21 \mathrm{I}: 4.73 \mathrm{cc}$. of $0.2 \mathrm{~N} \mathrm{NaOH}$ (using phenolphthalein).

Calc. for phostonic acid, $6.2 \mathrm{cc}$; allowing for $10 \%$ of barium as barium salt, $4.3 \mathrm{cc}$.

The acid decolorized permanganate solution only $1 / 7$ as fast as a sample of hydroxy-phosphonic acid. It did not form a crystalline aniline salt when treated with aniline in ether solution, a reaction very characteristic 
of the bydroxy-benzyl phosphoric acid. Only a non-crystallne rreterial was obtained which yielded no solid in alcohol or ether solution when seeded with the aniline salt of hydroxy-benzyl phosphonic acid.

\section{Conversion of the Phostonic Acid into the Hydroxy Phosphonic Acid.}

Two $\mathrm{g}$. of the impure phostonic acid described above was warmed on the steam-bath with $75 \mathrm{cc}$. of conc. hydrochloric acid until a clear solution was obtained. The solution was fittered to remove a slight amount of insoluble material. The filtrate was treated with dil. sulfuric acid until no further precipitate of barium sulfate formed. The mixture was filtered and evaporated to dryness. The crude hydroxy-benzyl phosphonic acid thus obtained was changed into its aniline salt as previously described. 2.2 g. of pure salt, m. p. $200^{\circ}$ to $201^{\circ}$, was thus obtained; this was $67 \%$ of the theoretical amount. Three $g$. of a sample of the barium sait of the phostonic acid was similarly treated and yielded $2.6 \mathrm{~g}$. of aniline salt, which was $96 \%$.

This hydrolysis of the phostonic acid to the hydroxy-phosphonic acid could be followed by determining the increase in acidity of a solution of the barium salt on boiling with water. $0.594 \mathrm{~g}$. of the salt (neutral to phenolphthalein) was boiled with $55 \mathrm{cc}$. of water under a returi condenser. At the end of $3 \frac{1}{2}$ hours the solution required 0.00029 equiva. lent of sodium hydroxide for neutralization; the hydrolysis was thus $36 \%$ complete. In similar experiments dil, hydrochloric acid was substituted for water and the net gain in acidity deternined. With a total acid concentration of $0.033 \mathrm{~N}$, the hydrolysis was $49 \%$ complete in 3 hours, and $67 \%$ in $x 6$ hours. With an acid concentration of $0 . x N$, in r hour $45 \%$ was converted; in 8 hours, $66 \%$. The reaction is evidently accelerated by the presence of the hydrogen ion.

\section{Reaction in Glacial Acetic Acid.}

In the first experiments with glacial acetic acid as a medium the aldehyde and acid were mixed together and the trichloride run in slowly from a dropping funnel. The reaction mixture was then allowed to stand at a definite temperature. Test samples were withdrawn from time to time and added to water. The amount of unreacted benzaldehyde was determined in the specially constructed pipet previously referred to. It was found that at temperatures below $25^{\circ}$ the reaction proceeded very slowly; at temperatures above $40^{\circ}$, on the other hand, the reaction seemed to proceed rapidly, but never more than $70 \%$ of the aldehyde was used up. This was undoubtedly due to the rapid reaction of the trichloride directly with the acetic acid at this temperature. The most favorable temperature was $30^{\circ}$ to $35^{\circ}$. Even under these conditions, however, about. $15 \%$ of the benzaldehyde was recovered unchanged.

The best results were obtained by first cautiously mixing the aldehyde 
and trichloride at $20^{\circ}$ to $40^{\circ}$, allowing the mixture to stand for one or two hours and then adding glacial acetic acid. The mixture was then kept between $30^{\circ}$ and $35^{\circ}$ and allowed to stand for one or two hours longer. At the end of this time the reaction was complete and all the aldehyde had reacted as shown by the absence of any oil when the mixture was poured into water. In all these experiments a $10 \%$ excess of trichloride was used and an amount of acetic acid equivalent to 3 or 4 mols. per mol. of aldehyde.

In the preparation of hydroxy-benzyl phosphonic acid by this method the reaction mixture is poured into water and evaporated to dryness. The syrup which is left deposits crystals of the acid on cooling. These may be freed from adhering syrup on a porous plate and recrystallized from dil. hydrochloric acid. However, the yield is low by this procedure because of the difficulty of handling the easily soluble phosphonic acid in the presence of a little phosphoric acid formed by a side reaction. The acid may be more conveniently isolated as the aniline salt.

A typical preparation of the hydroxy-benzyl phosphonic acid by this procedure follows. Ten $\mathrm{g}$. of benzaldehyde (one mol.) and $\mathrm{I}_{4} \mathrm{~g}$. of phosphorus trichloride (one mol. $+10 \%$ excess) were cautiously mixed in a flask fitted with a calcium chloride drying tube. After standing for one hour, I8 g. ( 3 mol.) of glacial acetic acid was added and the mixture allowed to stand for 3 hours. The temperature was kept between $25^{\circ}$ and $35^{\circ}$ during the entire experiment. The mixture was then poured into $200 \mathrm{cc}$. of water and evaporated to dryness on the steam-bath. The crude acid was dissolved in a little alcohol and the solution diluted with roo cc. of ether. Io $\mathrm{g}$. of aniline dissolved in ro cc. of ether was then added and the precipitate of the aniline salt filtered off. Yield, $18 \mathrm{~g}$., or $72 \%$. The isolation of the free acid from this salt has already been described.

The phostonic acid is formed directly in the reaction in glacial acetic acid but is slowly changed into the hydroxy-phosphonic acid on standing. The following experiments illustrate this. Ten g. of aldehyde, $13 \mathrm{~g}$. of trichloride, and $17 \mathrm{~g}$. of acetic acid were allowed to react as just described. The reaction mixture was then evaporated under diminished pressure at $50^{\circ}$ for 2 hours. A colorless gum was left. This gum was dissolved in a little water and treated with the calculated amount of a solution of barium hydroxide. The solution was made barely alkaline with ammonia and the white precipitate filtered, washed, and dried; yield, $9.5 \mathrm{~g}$., or $43 \%$. Tests on the salt showed that it was the barium salt of the phostonic acid. A similar experiment was carried out and the reaction mixture allowed to stand for 7 days before evaporation. Three $g$. of the barium salt was obtained which is only a $\mathrm{I} 3 \%$ yield. Hydrogen chloride accelerates this conversion of the phostonic acid into the hydroxy-phosphonic acidin acetic 
acid solution. A reaction was carried out in the usual way with acetic acid and the mixture then saturated with dry hydrogen chloride and allowed to stand overnight. The amount of barium salt obtained corresponded to only $16 \%$.

The mixture of acid chloride and anhydride obtained in the experiments with acetic anhydride was dissolved in acetic acid and the solution saturated with dry hydrogen chloride. After standing overnight, $53 \%$ of barium salt was obtained by the usual procedure. In similar experiments only $30 \%$ of salt was obtained after 3 days standing and only $22 \%$ after i I days. A sample of the original mixture of acid chloride and anhydride yielded $63 \%$ of the barium salt when treated airectly with water and barium hydroxide. It is evident that in glacial acetic acid the phosphorus oxygen ring is slowly opened by hydrogen chloride.

\section{Summary.}

I. Phosphorus trichloride forms an addition compound with benzaldehyde. The reaction is reversible and quantitative experiments show that the factors governing the equilibrium can be expressed in terms of the law of mass action.

2. The values obtained for the equilibrium constant vary from 6.9 to 8.3 the average is 7.4 .

3. The addition compound reacts with acetic anhydride forming acetyl chloride and a mixture of an acid anhydride and an acid chloride.

4. The structures of the anhydride and the chloride, and in turn that of the addition product, follow from their transformation by water into the inonobasic acid. This acid can be hydrolyzed to hydroxy-benzyl-phosphonic acid. It therefore, contains a 3 -membered carbon oxygen phosphorus ring; it is suggested that acids of this type be called phostonic acids.

5. Benzaldehyde, phosphorus trichloride and glacial acetic acid react to form acetyl chloride and the phostonic acid together with some hydroxy-phosphonic acid. Dilution with water and subsequent evaporation of the solution to dryness converts the phostonic acid into the hydroxyphosphonic acid. The latter acid can be converiently isolated it the form of an aniline salt.

Camarmar, Mass, 